\title{
Myalgic encephalomyelitis /chronic fatigue syndrome as a breakdown of homeostasis
}

\author{
David F Marks
}

Funding: The author(s) received no specific funding for this work.

Potential competing interests: The author(s) declared that no potential competing interests exist.

\begin{abstract}
ME/CFS a multi-system, complex illness disabling millions of people worldwide. A new theory holds that ME/CFS is caused by a breakdown of homeostasis. Homeostasis is a fundamental physiological principle that ensures equilibrium, stability and safety of the organism in a continuously changing and potentially life-threatening environment. A breakdown in homeostasis produces an aberrant state of disequilibrium with endocrinological and immunological changes consistent with the experienced disease symptoms.
\end{abstract}

ME/CFS a multi-system, and complex illness disabling millions of people worldwide. The Centers for Disease Control and Prevention (CDC) defines ME/CFS as follows:

People with ME/CFS are often not able to do their usual activities. At times, ME/CFS may confine them to bed. People with ME/CFS have overwhelming fatigue that is not improved by rest. ME/CFS may get worse after any activity, whether it's physical or mental. This symptom is known as post-exertional malaise (PEM). Other symptoms can include problems with sleep, thinking and concentrating, pain, and dizziness.

There is no agreed scientific explanation of the disorder, no treatment and no cure. The most popular theory has been that the condition is psychosomatic. However, the empirical evidence supporting this theory is based on correlational not causal research designs and, in my view, the theory is wrong and causes patient harm.

\section{Physiological Homeostasis}

The body uses three biological systems for the maintenance of equilibrium: the nervous system, the endocrine system and the immune system. The three systems communicate using electrical and chemical signals. They activate and deactivate tissues, organs and muscles to control and regulate the body, the emotions and the mind. The principal objective of the three systems is to preserve homeostasis. It is proposed that the complex multi-system disorder known as ME/CFS is caused by a breakdown of homeostasis. The 'Homeostasis Theory of ME/CFS' is supported by a substantial number of studies in the recent literature.

The three systems and their relationships to the brain and behaviour are illustrated in Figure 1. 


\section{Behaviour}

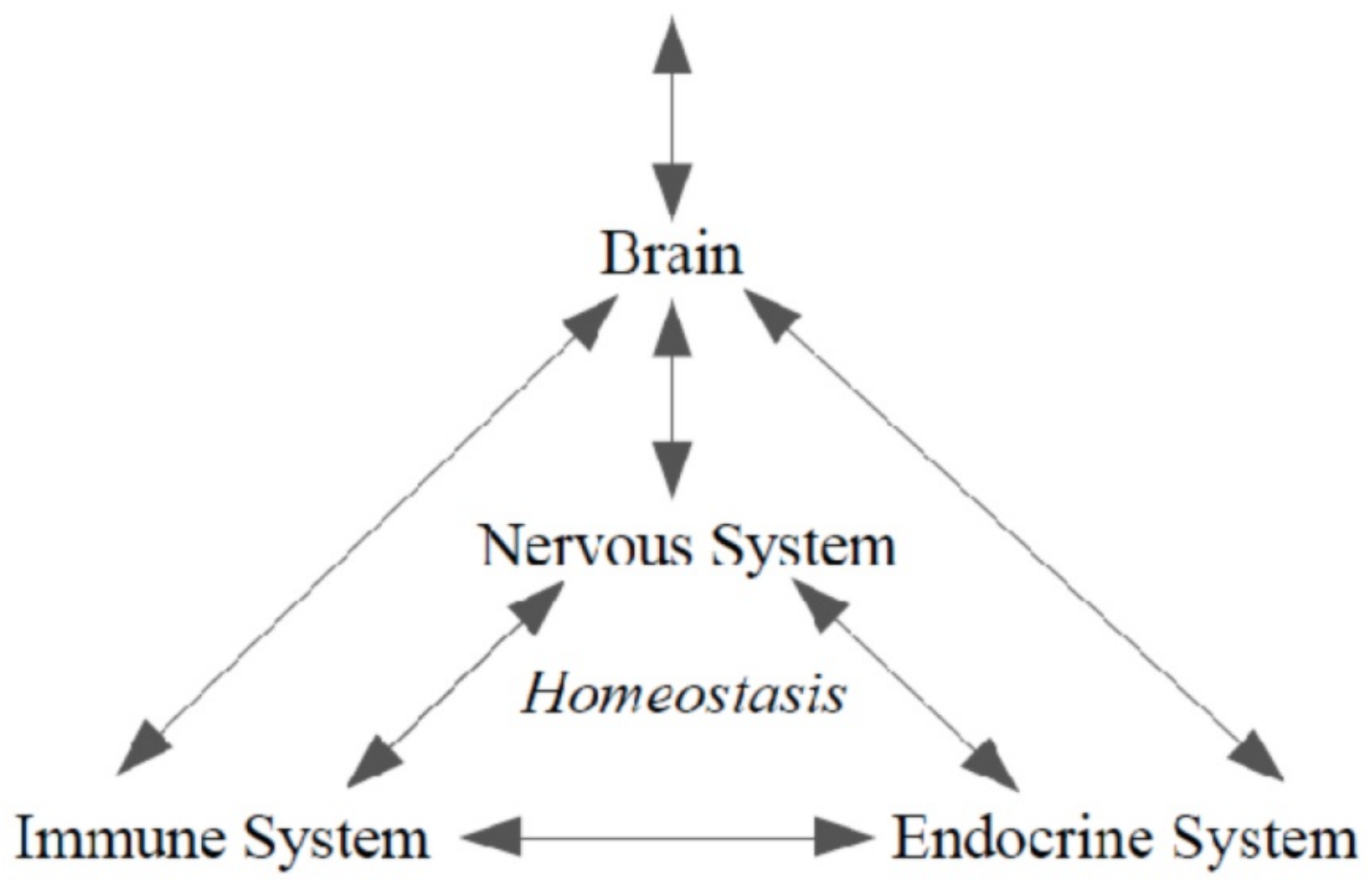

Figure 1 The nervous, endocrine and immune systems, and their relationships to brain and behaviour. Reproduced with permission from Marks, Murray and Estacio (2020, p. 26).

Continuous, reciprocal interaction between the brain, organs and gut is essential for smooth and efficient control and coordination of bodily functions, experience and behaviour. Endocrine substances directly affect the nervous and immune systems. The CNS innervates every organ and tissue of the immune system with reciprocal connections. The continuous interactions of the nervous, endocrine and immune systems was termed 'neuroimmunomodulation' by Spector and Korneva (1981). If a breakdown in homeostasis occurs, an individual is in serious trouble. This may precisely be the case in people with ME/CFS (pwME/CFS).

The chronic nature of ME/CFS suggests that its pathogenesis involves a disruption of homeostasis leading to chronic inflammation and immunological dysfunction. Biomarkers of inflammation and leaky gut syndrome as a possible result of microbiome disturbance and bacterial translocation have been reported (Shukla et al., 2015; Cortes Rivera et al., 2019; Mandarano et al., 2018; Sweetman et al., 2019). According to this theory, an aberrant state of homeostasis is the central cause of ME/CFS (Hatziagelaki et al., 2018; Nacul et al., 2020; Sweetman et al., 2019). To quote Sweetman et al. (2020):

"In people who develop ME/CFS, normal homeostatic processes in response to adverse insults may be replaced by aberrant responses leading to dysfunctional states. Thus, the predominantly neuro-immune manifestations, underlined by a hyper-metabolic state, that characterize early disease, may be followed by various processes leading to multi-systemic abnormalities and related symptoms. This abnormal state and the effects of a range of mediators such as products of 
oxidative and nitrosamine stress, may lead to progressive cell and metabolic dysfunction culminating in a hypometabolic state with low energy production. These processes do not seem to happen uniformly; although a spiraling of progressive inter-related and self-sustaining abnormalities may ensue, reversion to states of milder abnormalities is possible if the host is able to restate responses to improve homeostatic equilibrium."

In line with what is known about the typical course of the disease, it can reasonably be assumed that the hypothesized state of aberrant homeostasis can be aggravated by new stressors in the form of infection (Shepherd \& Chaudhuri, 2019), physical exertion, cognitive effort such as reading or solving mental puzzles, triggering a post-exertional malaise (PEM), comorbid conditions such as sleep disturbances (Chu, Valencia, Garvert \& Montoya, 2018) and other factors (Nacul et al., 2020). In people who happily do not develop ME/CFS or prolonged illness following an acute infection or other insult, external stressors initially cause short-term physiological changes but the usual state of homeostatic equilibrium that operated before the insult is quickly restored. Hatziagelaki et al. (2018) suggested that the aberrant state of homeostasis could be caused by inflammation in the hypothalamus (Figure 2).

\section{Hypothalamus}

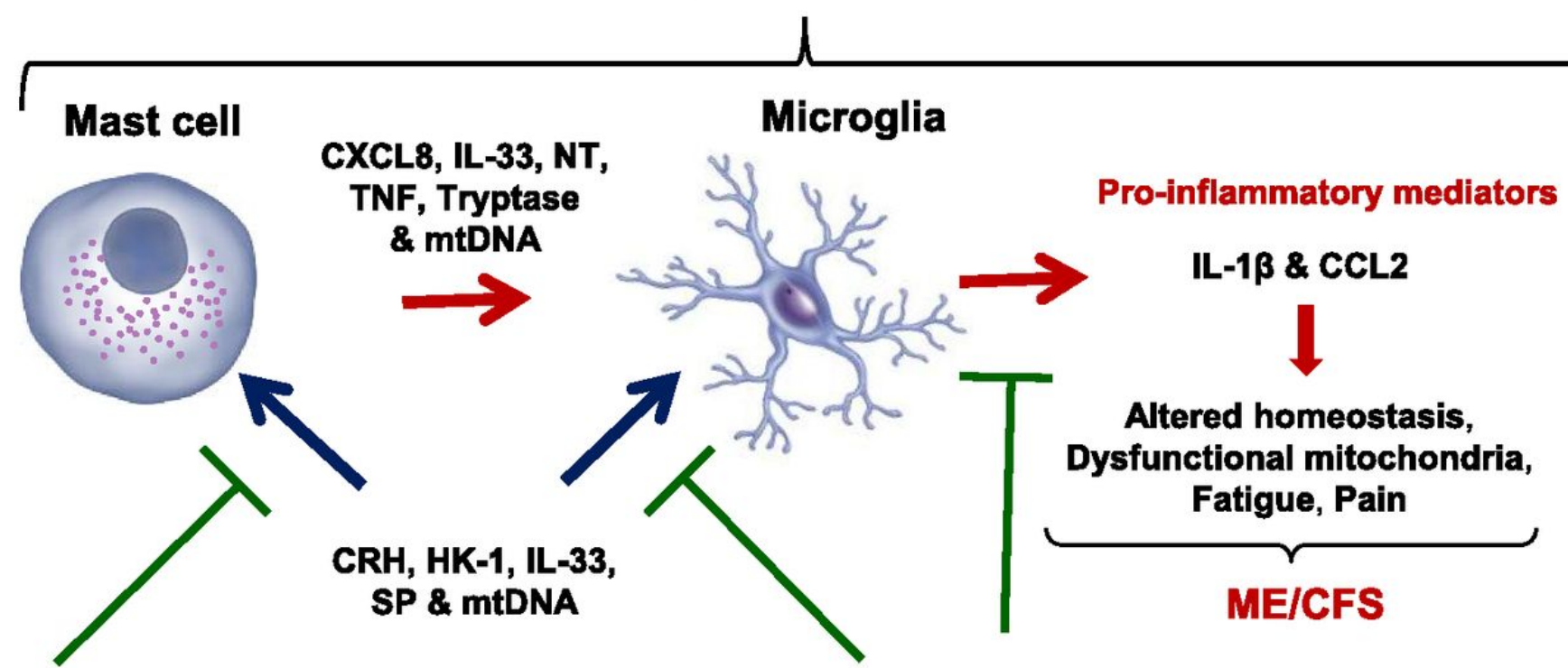

\section{Luteolin}

\section{Luteolin}

Figure 2. Diagrammatic representation of the proposed mast cell/microglia interactions in the hypothalamus contributing to the pathogenesis of ME/CFS, which could serve as targets for treatment. Hypothalamic mast cells are stimulated by stress-associated triggers such as CRH, HK-1, and SP, along with mtDNA and IL-33; some derive from the nasal cavity, while others may reach the area through a disrupted blood-brain barrier or through lymphatics. Stimulated mast cells then secrete molecules such as CXCL8, neurotensin (NT), TNF, tryptase, and mtDNA (CXCL), which activate microglia to secrete more inflammatory molecules (especially, IL-1 $\beta$, IL-6, and CXCL8) that further disrupt homeostasis, causing mitochondrial dysfunction and contributing to symptoms of ME/CFS. Luteolin could inhibit these processes at different steps, as shown. Reproduced from Hatziagelaki et al. (2018).

The Neuroimmune Hypothesis

Patients with ME/CFS often present with an acute onset of illness, with symptoms similar to an influenza infection, but these symptoms do not subside. Many of the symptoms are inflammatory, i.e. myalgia, arthralgia or joint pains, sore throat and tender lymphadenopathy. The immune system is a network of cells, tissues and organs that protects the body 
against disease or other potentially damaging foreign bodies. When properly functioning, the system identifies and attacks a variety of threats using billions of diverse antibodies, including viruses, bacteria and parasites, while distinguishing them from the body's own healthy tissue. For each type of invader the body needs a distinct antibody. Antibodies are made by B cells using a combination of 20,000 genes and an enzyme called 'RAG', which is a DNA shuffler. This enables the immune system to create a vast diversity of antibodies and respond to diseases it has never encountered before.

Inflammation is a critical defence response in our innate immune system wherein white blood cells protect us from infection by foreign organisms, bacteria and viruses. Inflammation occurs following infection or tissue damage when a rapid and complex series of reactions takes place to prevent tissue damage, isolate and destroy the infective organism, conserve and protect some micronutrients and activate the repair processes to restore normal functioning. Inflammation is a homeostatic process that is only intended to last a few days but, if it is continued indefinitely, there is a poor prognosis in many conditions. Inflammatory responses take precedence over normal body metabolism with the objective of restoring normality as quickly as possible.

Immune activation indicated by elevated levels of cytokines in pwME/CFS have been studied since 2001 Patarca, 2001). Recent studies have used immune system profiling to determine whether an abnormal profile of circulating cytokines could be identified in pwME/CFS and whether this profile correlated with disease severity and/or fatigue duration. In one study, serum cytokines of 192 pwME/CFS and 392 healthy controls were measured (Montoya et al., 2017). Seventeen cytokines showed a statistically significant upward linear trend that correlated with ME/CFS severity of which 13 were proinflammatory, which is likely to be a contributing factor to the symptoms.

Strawbridge, Sartor, Scott and Cleare (2019) carried out a systematic review and meta-analysis of 42 studies of circulating inflammatory proteins in pwCFS and non-affected control groups. Patients with CFS had significantly elevated tumour necrosis factor, interleukin-2, interleukin-4, transforming growth factor- $\beta$ and c-reactive protein. The authors concluded: "These data provide some support for an inflammatory component in CFS, although inconsistency of results indicates that inflammation is unlikely to be a primary feature in all those suffering from this disorder."

The generic cause of the abnormal inflammatory response in pwME/CFS is a breakdown in homoeostasis. The precise mechanism remains uncertain (Komoroff, 2019). However an inflammatory response is definitely present.

\section{The Microbiome Hypothesis}

The field of microbiome research studies the microbes within the gut and the effects of these microbes on the host's wellbeing. The microbiome is the community of symbiotic and pathogenic microorganisms, that live on the skin and genitals and in the nose, ears, mouth and gut. Microbes influence metabolism, immunity and behaviour. One mechanism appears to involve hormones because specific changes in hormone levels correlate with the presence of the gut microbiota. The microbiota produce and secrete hormones, respond to host hormones and regulate expression levels of host hormones. Hormones and the microbiome are linked to immune responses under both healthy conditions and autoimmune disease. There are many interconnections and the microbiome and hormones may work through shared pathways to affect the immune response (Neuman et al., 2015). 
Zhang et al. (2018) illustrate the homeostasis system's response to changes in diet and drug ingestions using the diagram below (Figure 3).

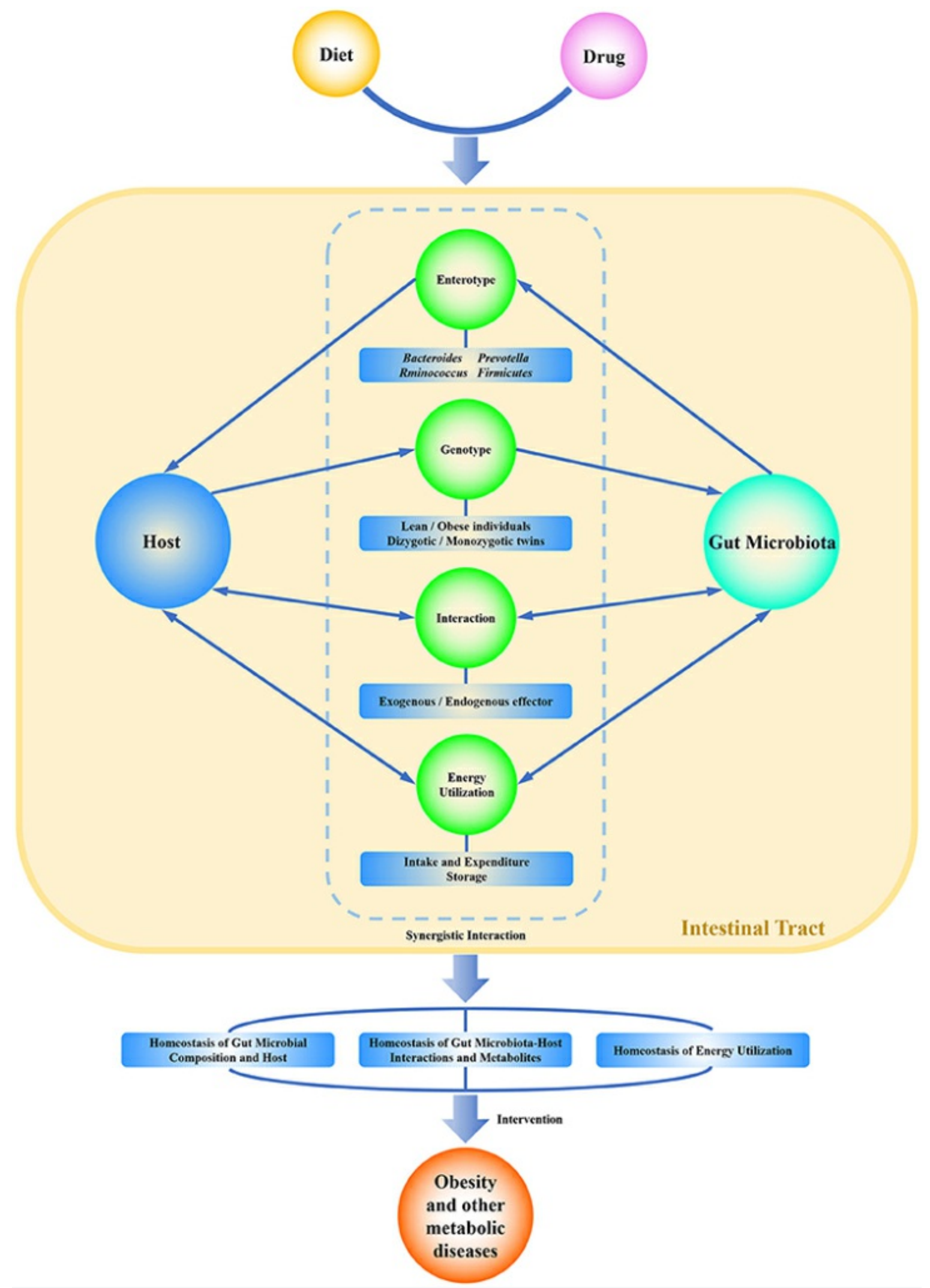

Figure 3. Graphical representation of the commensal homeostasis of gut microbiota-host under the intervention of diet and drugs. Diet and drugs can be digested, absorbed, and metabolized by the host and gut microbiota. The host and gut microbiota can interact synergistically to impact the physiological status of the human body. They not 


\begin{abstract}
only support themselves directly by using nutritional substrates but also impact each other indirectly by enterotype, genotype, metabolites, and related functions. Ultimately, the host and gut microbiota achieve a commensal homeostasis of composition, interactions, metabolites, and energy utilization. Reproduced from Zhang et al. (2018).
\end{abstract}

Organisms within the gut play a role in the early programming and response to stress. The gut contains $10^{3}-10^{14}$ microorganisms, ten times the number of cells in the human body, and contains $150-360$ times as many genes as our genome. That's a heck of a lot. When a pathogen such as Escherichia coli (E coli) enter the gut, the $\underline{H P A}$ can be activated. Stress can induce increased permeability of the gut, allowing bacteria and bacterial antigens to cross the epithelial barrier and activate a mucosal immune response, which in turn alters the composition of the microbiome and leads to an enhanced HPA drive.

Patients with irritable bowel syndrome and major depression show alterations of the HPA which are induced by increased gut permeability. In the case of irritable bowel syndrome, the increased permeability can respond to probiotic therapy. The gut microbiota play a role in regulating the HPA. Verdino (2017) cautiously concludes his review of the connection between gut health and emotional well-being as follows: “... it is crucial not to oversimplify the idea that nutritional intervention and a healthy gut will be the panacea for profound psychological difficulties. Severe mood and paralyzing anxiety disorders are not going to be cured with probiotic yogurt and prebiotic fiber, alone" (Verdino, 2017: 4).

The immune and neuroendocrine systems share a set of hormones and receptors. Glucocorticoids, such as corticosterone and cortisol, regulate inflammation levels and have effects both on the innate and adaptive immune responses. Additionally, vitamin D affects immune cell responses by enhancing antigen presentation. Moreover, sex hormones affect the immune response in numerous ways.

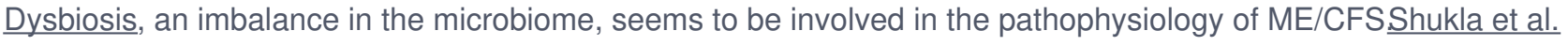
(2015) studied changes in the gut and plasma microbiome following exercise challenge in pwME/CFS. In exercise challenge, changes occurred in the amount of bacterial phyla in the gut in pwME/CFS that were not observed in healthy controls. Also, bacteria clearance from the blood was delayed in pwME/CFS following exercise. Altered gut microbiome and increased bacterial translocation following exercise thus appear to play a role in ME/CFS that may account for the post-exertional malaise experienced by many pwME/CFS.

\title{
Mitochondrial Failure
}

Mitochondrial failure is another part of the jigsaw puzzle posed by ME/CFS (Myhill et al. 2009). Mitochondria generate cellular energy by manufacturing ATP (adenosine triphosphate). Infections with pathogens, including viruses, bacteria, parasites and toxins can cause changes in the function of mitochondria and deplete energy metabolism (Figure 4). Metabolomics, the systematic identification of metabolic products (the metabolome) of a biological system is revealing a possible chemical pattern of chemical abnormalities or metabolite abnormalities in pwME/CFS. Myhill, Booth and McLaren-Howard (2009) developed interventions based on the biochemistry of the illness, specifically the function of mitochondria in producing ATP, the energy currency for all body functions, and recycling ADP to replenish the ATP supply as needed. Patients attending a private medical practice specializing in ME/CFS were diagnosed using the Centers for 
Disease Control criteria. A correlation was obtained between the degree of mitochondrial dysfunction and the severity of illness $(\mathrm{P}<0.001)$. Only 1 of 71 patients overlapped the normal region.

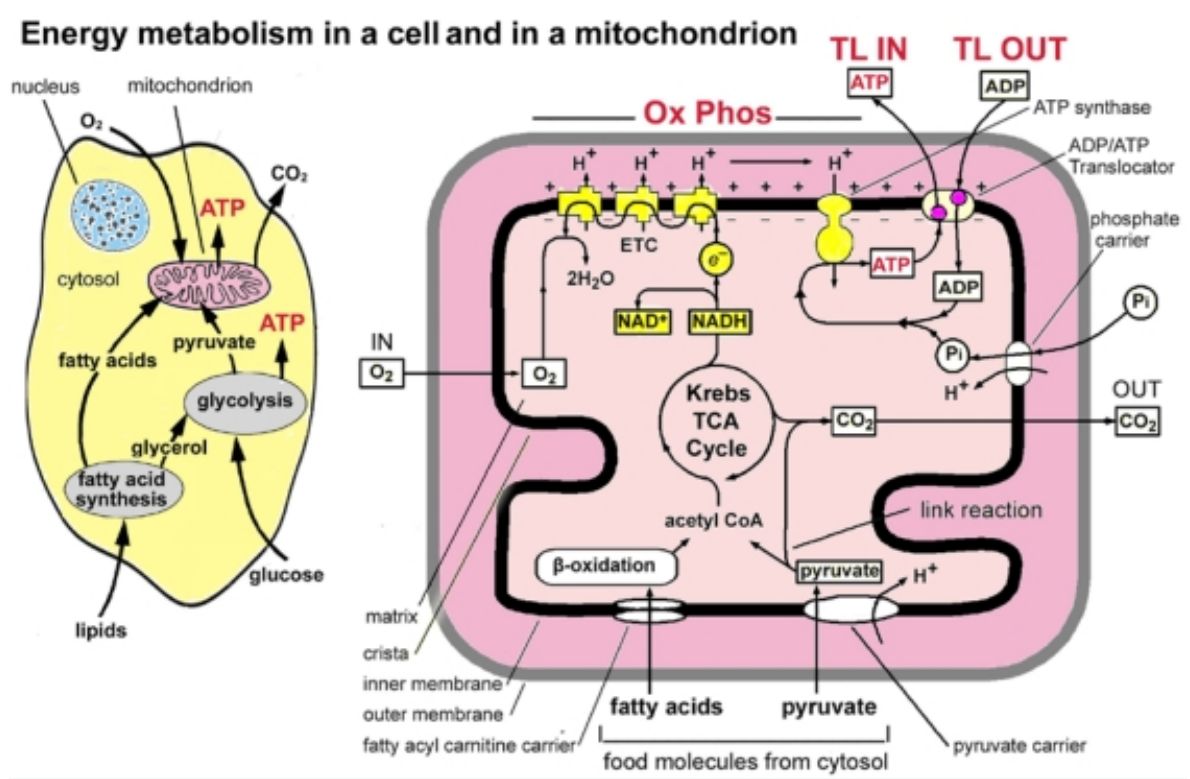

Figure 4. Main stages and location of energy metabolism in a human cell (left), and simplified details of a mitochondrion showing the main metabolic cycles and the oxidative phosphorylation respiratory chain (right). The outer mitochondrial membrane is highly permeable whereas the inner membrane is permeable only to water and gases. Special carrier and Translocator proteins pass reactants through it. At the top are the proteins involved in the respiratory electron transfer chain (ETC) and in the transfer of ATP and ADP between the cytosol and mitochondrion. ADP and $P_{\mathrm{i}}$ are combined by ATP synthase to make ATP. The ADP/ATP Translocator opens OUT to transfer ADP into the matrix and opens IN to transfer ATP to the cytosol. Nicotinamide adenine dinucleotide plays a key role in its oxidised form $\mathrm{NAD}^{+}$and its reduced form $\mathrm{NADH}+\mathrm{H}^{+}$in carrying and transferring protons $\left(\mathrm{H}^{+}\right)$and electrons $\left(e^{-}\right)$. Reproduced from Myhill et al. 2009.

The authors concluded that "the "ATP profile" test is a powerful diagnostic tool and can differentiate patients who have fatigue and other symptoms as a result of energy wastage by stress and psychological factors from those who have insufficient energy due to cellular respiration dysfunction. The individual factors indicate which remedial actions, in the form of dietary supplements, drugs and detoxification, are most likely to be of benefit, and what further tests should be carried out" (Myhill et al., 2009).

Morris and Maes (2014) support Myhill et al.'s approach in arguing that mitochondrial dysfunctions in the form of lowered ATP production may play a role in the onset of ME/CFS symptoms and help to explain in part the central metabolic abnormalities observed in ME/CFS, e.g. glucose hypometabolism and cerebral hypoperfusion.

The results of a study by Sweetman et al. (2020) also "support a model of deficient ATP production in ME/CFS, compensated for by upregulation of immediate pathways upstream of Complex $V$ that would suggest an elevation of oxidative stress." 


\section{Conclusion}

The specific causal mechanism for ME/CFS remains uncertain. Whatever it turns out to be, homeostasis breakdown appears likely to be the underlying problem.

\section{REFERENCES}

Centers for Disease Control and Prevention (2021). https://www.cdc.gov/mecfs/index.html\#: :text=Myalgic\%20encephalomyelitis\%2Fchronic\%20fatigue\%20syndrome\%20(ME\%2FCFS)\%20is,sever e\%20fatigue\%20and\%20sleep\%20problems.

Chu, L., Valencia, I. J., Garvert, D. W., \& Montoya, J. G. (2018). Deconstructing post-exertional malaise in myalgic encephalomyelitis/chronic fatigue syndrome: A patient-centered, cross-sectional survey. PloS one, 13(6), e0197811.

Cortes Rivera, M., Mastronardi, C., Silva-Aldana, C. T., Arcos-Burgos, M., \& Lidbury, B. A. (2019). Myalgic encephalomyelitis/chronic fatigue syndrome: a comprehensive review. Diagnostics, 9(3), 91.

Hatziagelaki, E., Adamaki, M., Tsilioni, I., Dimitriadis, G., \& Theoharides, T. C. (2018). Myalgic encephalomyelitis/chronic fatigue syndrome - metabolic disease or disturbed homeostasis due to focal inflammation in the hypothalamus?. Journal of Pharmacology and Experimental Therapeutics, 367(1), 155-167.

Komaroff, A. L. (2019). Advances in understanding the pathophysiology of chronic fatigue syndrome.Jama, 322(6), 499500.

Mandarano, A. H., Giloteaux, L., Keller, B. A., Levine, S. M., \& Hanson, M. R. (2018). Eukaryotes in the gut microbiota in myalgic encephalomyelitis/chronic fatigue syndrome. PeerJ, 6, e4282.

Marks, D.F., Murray, M. and Estacio, E.V. (2020) Health psychology. Theory, research \& practice. ( $6^{\text {th }}$ ed). London: Sage Publications.

Morris, G., \& Maes, M. (2014). Mitochondrial dysfunctions in myalgic encephalomyelitis/chronic fatigue syndrome explained by activated immuno-inflammatory, oxidative and nitrosative stress pathways. Metabolic brain disease, 29(1), 19-36.

Myhill, S., Booth, N. E., \& McLaren-Howard, J. (2009). Chronic fatigue syndrome and mitochondrial dysfunction. International journal of clinical and experimental medicine, 2(1), 1.

Nacul, L., O'Boyle, S., Palla, L., Nacul, F. E., Mudie, K., Kingdon, C. C., ... \& Lacerda, E. M. (2020). How Myalgic Encephalomyelitis/Chronic Fatigue Syndrome (ME/CFS) Progresses: The Natural History of ME/CFS. Frontiers in neurology, 11, 826.

Neuman, H., Debelius, J. W., Knight, R., \& Koren, O. (2015). Microbial endocrinology: the interplay between the microbiota and the endocrine system. FEMS microbiology reviews, 39(4), 509-521. 
Patarca, R. (2001). Cytokines and chronic fatigue syndrome. Annals of the New York Academy of Sciences 933(1), 185200.

Shepherd, D. C., \& Chaudhuri, D. A. (2019).ME/CFS/PVFS: an exploration of the key clinical issues ME Association.

Shukla, S. K., Cook, D., Meyer, J., Vernon, S. D., Le, T., Clevidence, D., ... \& Frank, D. N. (2015). Changes in gut and plasma microbiome following exercise challenge in myalgic encephalomyelitis/chronic fatigue syndrome (ME/CFS). PLOS One, 10(12), e0145453.

Spector, N. H., \& Korneva, E. A. (1981). Neurophysiology, immunophysiology, and neuroimmunomodulation (Vol. 449). Academic Press, New York.

Strawbridge, R., Sartor, M. L., Scott, F., \& Cleare, A. J. (2019). Inflammatory proteins are altered in chronic fatigue syndrome—a systematic review and meta-analysis. Neuroscience \& Biobehavioral Reviews, 107, 69-83.

Sweetman, E., Noble, A., Edgar, C., Mackay, A., Helliwell, A., Vallings, R., ... \& Tate, W. (2019). Current research provides insight into the biological basis and diagnostic potential for myalgic encephalomyelitis/chronic fatigue syndrome (ME/CFS). Diagnostics, 9(3), 73.

Sweetman, E., Kleffmann, T., Edgar, C., de Lange, M., Vallings, R., \& Tate, W. (2020). A SWATH-MS analysis of Myalgic Encephalomyelitis/Chronic Fatigue Syndrome peripheral blood mononuclear cell proteomes reveals mitochondrial dysfunction. Journal of translational medicine, 18(1), 1-18.

Verdino, J. (2017). The third tier in treatment: Attending to the growing connection between gut health and emotional wellbeing. Health psychology open, 4(2), 2055102917724335.

Zhang, Y., Sowers, J. R., \& Ren, J. (2018). Targeting autophagy in obesity: from pathophysiology to management.Nature Reviews Endocrinology, 14(6), 356-376. 